\title{
FLT-PET for the assessment of systemic sarcoidosis including cardiac and CNS involvement: a prospective study with comparison to FDG-PET
}

Patrick Martineau ${ }^{10}$, Matthieu Pelletier-Galarneau ${ }^{2,3}$, Daniel Juneau ${ }^{4,5}$, Eugene Leung ${ }^{6}$, Pablo Nery ${ }^{7}$, Rob deKemp ${ }^{5}$, Rob Beanlands ${ }^{5 \dagger}$ and David Birnie ${ }^{7 *+}$

\begin{abstract}
Background: 2-deoxy-2-[18F]fluoro-D-glucose's (FDG) biodistribution limits the evaluation of cardiac sarcoidosis (CS) and neurosarcoidosis (NS). While protocols for cardiac suppression exist, they can be inconvenient for patients and lead to incomplete cardiac suppression in many cases. Furthermore, FDG PET is limited in the detection of neurosarcoidosis due to an inability to suppress high level of physiological uptake within the brain. $3^{\prime}$-deoxy- $3^{\prime}-\left[{ }^{18} \mathrm{~F}\right]$ fluorothymidine (FLT) has been shown to accumulate in sarcoidosis lesions and this tracer lacks significant physiological myocardial and brain uptake, suggesting that this tracer may be useful for the assessment of sarcoidosis, including CS and NS, without the need for patient preparation. This prospective pilot study examined the performance of FLT vs FDG PET for systemic sarcoidosis, including cardiac and neural involvement.
\end{abstract}

Materials and methods: Fourteen subjects with sarcoidosis were prospectively recruited and imaged with FDG- and FLT-PET. Two blinded, experienced readers independently reviewed the FLT-PET and FDG-PET images. Lesion distribution was compared between FLT and FDG. Agreement between FLT- and FDG-PET was determined using Cohen's kappa and the intra-class correlation coefficient. Inter-observer variability of FLT and FDG-PET was assessed.

Results: Twelve subjects had CS as per Heart Rhythm Society criteria and 1 had NS. FLT-PET was positive in 12 (86\%), and FDG-PET in 11 (79\%), with cardiac uptake present in $6(50 \%)$ and 7 (58\%) of subjects with CS, respectively. The subject with NS demonstrated uptake on both FLT and FDG-PET, with more lesions on FLT. There were no significant differences in the anatomical distribution of lesions between FLT and FDG. SUVs were significantly $(p<0.001)$ higher for FDG than FLT (5.8 \pm 3.0 vs $2.3 \pm 1.1$, respectively), but not ( $p=0.90)$ after adjusting for blood pool activity $(2.8 \pm 1.4$ vs $2.8 \pm 1.1$, respectively). Agreement between FLT- and FDG-PET was good to excellent for the diagnosis of sarcoidosis, lung involvement, CS, and NS ( $K=0.76,0.69,0.86$, and 1.0 , respectively). Inter-observer agreement for FLT was excellent for diagnosing sarcoidosis, CS and NS ( $K=0.81,0.85$, and 1.0 , respectively) and comparable to that of FDG.

Conclusions: FLT-PET may be useful for the assessment of systemic sarcoidosis, as well as cardiac and neural involvement.

\footnotetext{
*Correspondence: dbirnie@ottawaheart.ca

${ }^{\dagger}$ Rob Beanlands and David Birnie are co-senior authors of this work

${ }^{7}$ Arrhythmia Service, Division of Cardiology, Department of Medicine, University of Ottawa Heart Institute, 40 Ruskin Street - Room H 1285,

Ottawa, ON K1Y 4W7, Canada

Full list of author information is available at the end of the article
} original author(s) and the source, provide a link to the Creative Commons licence, and indicate if changes were made. The images or other third party material in this article are included in the article's Creative Commons licence, unless indicated otherwise in a credit line to the material. If material is not included in the article's Creative Commons licence and your intended use is not permitted by statutory regulation or exceeds the permitted use, you will need to obtain permission directly from the copyright holder. To view a copy of this licence, visit http://creativecommons.org/licenses/by/4.0/. 
Keywords: Sarcoidosis, Neurosarcoidosis, Cardiac sarcoidosis, Fluorothymidine, FLT-PET, FDG-PET

\section{Introduction}

The last few years have seen an increased recognition of the importance in the detection of cardiac sarcoidosis (CS), accounting for up to $85 \%$ [1] of sarcoid deaths. In addition, neurosarcoidosis (NS) is associated with a $10 \%$ mortality rate [2] and present in $5-15 \%$ of cases [3]. For both NS and CS, post-mortem studies have revealed that only a fraction of cases are diagnosed antemortem with up to $39 \%$ and $50 \%$ of cases of CS and NS detected on autopsy [4].

2-deoxy-2- $\left[{ }^{18} \mathrm{~F}\right]$ fluoro-D-glucose (FDG)-PET is useful in diagnosing and assessing the extent of active sarcoidosis, due to its ability to image inflammation. FDG-PET has been shown to be useful in identifying appropriate biopsy sites in patients with sarcoidosis as affected lymph nodes are often morphologically unremarkable and undetectable by other imaging modalities [5]. Furthermore, the degree of FDG uptake, particularly within lung parenchyma, has been shown to serve as an effective therapeutic target [6] with the degree of lung uptake decreasing following the initiation of therapy [7]; however, despite a near perfect sensitivity of $96-100 \%$ for pulmonary disease [8], the diagnostic accuracy for cardiac involvement is lower, with one meta-analysis reporting a sensitivity/specificity of $89 \% / 78 \%$ [9]. Furthermore, extensive patient preparation is required to suppress physiological FDG uptake and non-diagnostic or false positive studies due to suboptimal suppression can be seen in $10-20 \%$ of cases [10]. The role of FDG-PET in assessing NS remains largely unstudied.

$3^{\prime}$-deoxy- $3^{\prime}-\left[{ }^{18} \mathrm{~F}\right]$ fluorothymidine (FLT)-PET has recently been shown to be useful in the assessment of CS without requiring extensive pre-imaging preparation $[11,12]$. This prospective study investigated the use of FLT-PET for the evaluation of sarcoidosis, including myocardial and neural involvement, with comparison to FDG-PET.

\section{Methods}

\section{Subjects}

Subjects with tissue-proven sarcoidosis scheduled for a whole-body FDG-PET, perfusion PET and dedicated cardiac FDG-PET for the assessment of CS were considered for enrollment. Exclusion criteria included breastfeeding, pregnancy, claustrophobia, inability to lie still in a supine position, unwillingness or inability to provide informed consent, age $<18$ years, or active malignancy. The results of other relevant imaging studies, histopathology findings, and the subject's clinical histories were collected. All participants were subsequently imaged with FLT-PET (wholebody and ECG-gated cardiac images) within 2 weeks of FDG-PET imaging. Subject treatment was neither initiated nor changed in the interval between FDG and FLT imaging. This prospective single-center study was approved by the institutional research ethics board and all participants provided signed informed consent.

\section{Subject preparation and imaging protocol}

Imaging studies were performed in the following order: (1) cardiac rest perfusion scan; (2) whole-body FDG scan; (3) ECG-gated cardiac FDG scan. FLT studies were acquired in the following order: (1) whole-body FLT scan; (2) ECGgated cardiac FLT scan. All images were acquired in 3D mode and reconstructed using an iterative technique. PET images were performed on a Discovery 690 or 600 PET/CT scanner (GE Healthcare, Waukesha, WI). FLT scans were performed within 14 days of perfusion and FDG scans.

\section{Perfusion PET}

Following administration of either ${ }^{82} \mathrm{Rb}(10 \mathrm{MBq} / \mathrm{kg})$ or $\left[{ }^{13} \mathrm{~N}\right] \mathrm{NH}_{3}(3-5 \mathrm{MBq} / \mathrm{kg})$, subjects underwent rest ECGgated perfusion PET, with a low-dose CT for localization and attenuation correction. Rest perfusion images were reviewed using the standard 17-segment model [13] and a 5 -point grading system $(0=$ normal radiotracer uptake, $1=$ mildly reduced, $2=$ moderately reduced, $3=$ severely reduced, $4=$ absence of uptake).

\section{FDG-PET}

Subjects were instructed to follow a low-carbohydrate, high fat, protein-permitted diet (subjects were provided with detailed dietary instructions) followed by a fast for at least $12 \mathrm{~h}$. Low-dose unfractionated heparin was intravenously injected prior to FDG administration in participants without contraindication [10]; $5 \mathrm{MBq} / \mathrm{kg}$ of FDG was injected intravenously followed by an uptake phase of $60 \mathrm{~min}$ and whole-body imaging. A low-dose, non-contrast CT scan from the femoral region to the head was performed for attenuation correction; $90 \mathrm{~min}$ after FDG injection, a 20-min ECG-gated cardiac acquisition was performed.

\section{FLT-PET}

For FLT-PET imaging, participants were instructed to not eat or drink anything except water for six hours prior to imaging. No other preparation was specified.

Intravenous injection of $370 \mathrm{MBq}$ of FLT was followed by an uptake phase of $60 \mathrm{~min}$. The FLT-PET imaging was 
otherwise performed in an identical fashion to the FDGPET acquisition.

\section{Image interpretation}

Images were anonymized and reviewed in random order by two experienced nuclear medicine physicians (PM and DJ) blinded to any identifying information using HybridViewer PET-CT fusion software (Hermes Medical Solutions). CT findings were reviewed and used for anatomic localization and characterization of PET findings. Perfusion images and dedicated cardiac DG-PET and FLT-PET were reviewing using 4DM (INVIA Medical Imaging Solutions). Regions of interest (ROIs) were defined as spheres $1 \mathrm{~cm}$ in diameter. All reported SUVs consisted of $\mathrm{SUV}_{\mathrm{Max}}$ measured within an ROI. Blood pool SUVs were measured in a similar fashion using an ROI within the ascending thoracic aorta. Individual lesions were defined as foci of radiotracer uptake significantly greater than background and visually distinct from the normal biodistribution of the radiotracer. Active sarcoidosis was defined as uptake of either FDG or FLT in lesions consistent with sarcoidosis. A third reader resolved differences in interpretation.

\section{Reference standard}

The gold standard for sarcoidosis was tissue diagnosis while the gold standard for cardiac involvement consisted of the Heart Rhythm Society criteria [14]. Clinical diagnosis was considered the gold standard for NS.

\section{Statistical analysis}

Descriptive statistics are presented as the number (percent) for categorical variables and mean ( \pm standard deviation) for continuous variables. Normality was tested using the Shapiro-Wilk test. Differences between groups were compared using Student's paired samples $t$ test. Kappa $(\kappa)$ statistics were calculated for inter-observer agreement of categorical findings on FLT- and FDGPET scans while continuous variables were compared with the intra-class correlation coefficient (ICC) using a two-way random effects, absolute agreement, single rater model [15]. Interpretation of concordance measures was in keeping with the guidelines of Cicchetti et al. [16]. A $p$ value of $<0.05$ was considered statistically significant. All analyses were performed using SPSS v20 (IBM Corp. Released 2011. IBM SPSS Statistics for Windows, Version 20.0. Armonk, NY: IBM Corp).

\section{RESULTS}

\section{Baseline demographics}

Fourteen subjects were recruited between January $10^{\text {th }}$, 2017 and November $15^{\text {th }}$, 2017. Subject characteristics are shown in Table 1. Extra-cardiac sarcoidosis was confirmed in all subjects by lymph node tissue sampling. 12 (86\%) were diagnosed with CS, in accordance with the Heart Rhythm Society (HRS) criteria [14]. All subjects positive for CS had a histological diagnosis of extracardiac sarcoidosis, in addition to an appropriate cardiac presentation and/or cardiac MRI findings compatible with CS. Subjects with CS were diagnosed independently of the PET findings in this study (Table 1). 1 (7\%) subject had previously diagnosed NS.

\section{Imaging \\ Whole-body findings}

Image quality for all PET studies was excellent. Of the 14 subjects, 12 (86\%) showed FLT and 11 (79\%) FDG uptake compatible with active sarcoidosis. A single subject (\#8) demonstrated mild uptake (SUV 1.6 compared to a blood pool SUV of 1.4) in a few mediastinal lymph nodes on FLT, with no other uptake and without uptake on the corresponding FDG study. This subject was receiving treatment with methotrexate at the time of imaging. The distribution of lesions is shown in Table 2 with the most commonly affected sites consisting of thoracic lymph nodes ( $71 \%$ of FLT and FDG cases) and cervical lymph nodes (50\% of FLT and 57\% of FDG cases). Comparison of the anatomical distribution of lesions revealed no statistically significant differences (Table 2).

Figure 1 shows an example of whole-body FLT and FDG studies in the same subject with sarcoidosis and evidence of active CS and NS. Agreement between FLT- and FDG-PET for the assessment of individual sites varied but was highest for cardiac $[K=0.86(95 \%$ CI $0.59-1.0)]$ and CNS (spinal/paraspinal) $[\kappa=1.0(95 \%$ CI $1.0-1.0)]$ involvement (Table 2). Agreement for the involvement of lung parenchyma was good $[\kappa=0.69(95 \%$ CI $0.29-1.0)]$ while the agreement for the total number of affected sites was excellent $[\mathrm{ICC}=0.78(95 \% 0.45-0.93)]$. Overall, agreement between FLT- and FDG-PET for the diagnosis of active sarcoidosis was excellent $(\kappa=0.76(95 \%$ CI $0.32-1.0)$, Table 3). Inter-observer agreement for active sarcoidosis was excellent for both FLT and FDG $(\kappa=0.81$ (95\% CI $0.46-1.0)$ vs $\kappa=1.0$ (95\% CI $1.0-1.0$, respectively), Additional file 1: Table S1).

Mean SUV of sarcoidosis lesions differed significantly $(p<0.001)$ between FLT and FDG $(2.3 \pm 1.1$ and $5.8 \pm 3.0$, respectively); however, the difference was no longer significant $(p=0.90)$ after adjusting for blood pool activity (FLT, FDG: $2.8 \pm 1.1,2.8 \pm 1.4$ ). Blood pool activity was significantly less $(p<0.0001)$ for FLT than FDG $(1.2 \pm 0.2$, $2.4 \pm 0.4$, respectively). Linear regression was used to compare the blood pool adjusted SUV for FLT and FDG on a lesion-per-lesion level, for a total of 61 paired lesions (Fig. 2). The resultant equation is shown in Fig. 2 and was associated with an $\mathrm{R}$ of 0.51 . 
Table 1 Subject baseline characteristics

\begin{tabular}{|c|c|c|c|c|c|c|c|c|c|}
\hline Subject \# & Age (yrs) & Gender & Ethnicity & $\begin{array}{l}\text { Positive } \\
\text { for CS } \\
\text { by HRS } \\
\text { criteria? }\end{array}$ & $\begin{array}{l}\text { Cardiac } \\
\text { presentation }\end{array}$ & $\begin{array}{l}\text { Biopsy } \\
\text { proven } \\
\text { extra- } \\
\text { cardiac } \\
\text { sarcoidosis? }\end{array}$ & $\begin{array}{l}\text { Initial assessment } \\
\text { or reassessment? }\end{array}$ & $\begin{array}{l}\text { On active } \\
\text { treatment } \\
\text { at the time } \\
\text { of current } \\
\text { imaging? }\end{array}$ & $\begin{array}{l}\text { MRI findings } \\
\text { compatible } \\
\text { with CS? }\end{array}$ \\
\hline 1 & 44 & Male & Caucasian & Yes & $\begin{array}{l}\text { Bifascicular } \\
\text { block }\end{array}$ & Yes & Reassessment & $\begin{array}{l}\text { Yes, metho- } \\
\text { trexate/ } \\
\text { prednisone }\end{array}$ & Yes \\
\hline 2 & 64 & Male & Caucasian & No & $\begin{array}{l}\text { Cardiomyo- } \\
\text { pathy }\end{array}$ & Yes & Initial & No & No \\
\hline 3 & 49 & Male & Caucasian & Yes & $\begin{array}{l}\text { Complete AV } \\
\text { block }\end{array}$ & Yes & Initial & No & Yes \\
\hline 4 & 49 & Female & $\begin{array}{l}\text { African-Amer- } \\
\text { ican }\end{array}$ & Yes & $\begin{array}{l}\text { Complete AV } \\
\text { block }\end{array}$ & Yes & Initial & No & No \\
\hline 5 & 57 & Male & Caucasian & Yes & $\begin{array}{l}\text { Complete AV } \\
\text { block }\end{array}$ & Yes & Reassessment & No & Not performed \\
\hline 6 & 56 & Male & Caucasian & Yes & $\begin{array}{l}\text { Cardiomyo- } \\
\text { pathy }\end{array}$ & Yes & Reassessment & No & Not performed \\
\hline 7 & 54 & Female & Caucasian & No & None & Yes & Initial & No & No \\
\hline 8 & 66 & Male & Caucasian & Yes & $\begin{array}{l}\text { Complete AV } \\
\text { block }\end{array}$ & Yes & Reassessment & $\begin{array}{l}\text { Yes, metho- } \\
\text { trexate }\end{array}$ & Yes \\
\hline 9 & 68 & Male & Caucasian & Yes & None & Yes & Initial & No & Yes \\
\hline 10 & 57 & Male & Caucasian & Yes & $\begin{array}{l}\text { Ventricular } \\
\text { tachycardia }\end{array}$ & Yes & Reassessment & $\begin{array}{l}\text { Yes, pred- } \\
\text { nisone }\end{array}$ & Yes \\
\hline 11 & 54 & Female & Caucasian & Yes & $\begin{array}{l}\text { Complete AV } \\
\text { block }\end{array}$ & Yes & Initial & No & No \\
\hline 12 & 57 & Male & Caucasian & Yes & $\begin{array}{l}\text { Complete AV } \\
\text { block }\end{array}$ & Yes & Initial & No & Yes \\
\hline 13 & 58 & Female & Asian & Yes & $\begin{array}{l}\text { Complete AV } \\
\text { block }\end{array}$ & Yes & Initial & No & Yes \\
\hline 14 & 55 & Female & Asian & Yes & $\begin{array}{l}\text { Complete AV } \\
\text { block }\end{array}$ & Yes & Initial & No & Not performed \\
\hline
\end{tabular}

\section{Cardiac PET findings}

Additional details of the cardiac findings have been published separately [12]. 12 (86\%) subjects underwent perfusion PET with $\left[{ }^{13} \mathrm{~N}\right] \mathrm{NH}_{3}$ and $2(14 \%)$ with ${ }^{82} \mathrm{Rb}$. All cardiac PET studies were considered diagnostic. Of the 12 subjects positive by HRS criteria, 7 (58\%) showed myocardial FDG uptake consistent with active sarcoidosis, while myocardial FLT uptake was present in 6 (50\%). A single subject (\#10) demonstrated evidence of active CS on FDG without corresponding FLT activity (Fig. 3). In this subject, abnormal FDG uptake was limited to a single cardiac segment with an SUV of 3.5 (blood pool 2.6). This subject was receiving treatment with prednisone at the time of imaging and findings of extra-cardiac sarcoidosis were present on both the FLT and FDG studies.

An example of a perfusion PET, dedicated cardiac FDG-, and FLT-PET study in a subject with active CS is shown in Fig. 4 while Fig. 5 shows the relationship between cardiac findings and mediastinal uptake for FLT and FDG. All subjects with evidence of active CS had left ventricular (LV) uptake, while 4 and 2 of these had right ventricular (RV) uptake on FDG and FLT, respectively. None of our subjects had findings suggestive of isolated CS. One subject demonstrated isolated lateral wall myocardial FDG uptake (Fig. 6) without corresponding FLT uptake or perfusion deficits and was considered negative for active CS. The cardiac FDG findings in this subject were classified as negative. A summary of cardiac findings is shown in Table 4.

Concordance analysis of the cardiac findings in those subjects that satisfied the HRS criteria is shown in Table 5. Agreement between FLT and FDG for left and right ventricle involvement was excellent and fair, respectively, [left: $\kappa=0.86$ (95\% CI 0.59-1.0); right: $\kappa=0.59$ (95\% CI 0.082-1.0)]. When comparing the number of affected segments on FLT and FDG, the ICC was excellent overall $[\mathrm{ICC}=0.81(95 \% \mathrm{CI} 0.49-0.94)]$, as well as in the left anterior descending and right coronary artery territories (0.81 (95\% CI 0.51-0.94) and 0.84 (95\% CI 0.56-0.95), respectively). Agreement was fair [0.54 (95\% CI 0.03-0.83)] in the left circumflex territory. Agreement 
Table 2 Anatomical distribution of sarcoidosis lesions on FLT- and FDG-PET $(N=14)$

\begin{tabular}{|c|c|c|c|c|}
\hline Anatomical site & FLT-PET & FDG-PET & $p$ value & Agreement $^{\dagger}$ \\
\hline \multicolumn{5}{|l|}{ Lymph nodes } \\
\hline Thoracic & $10 / 14(71 \%)$ & $10 / 14(71 \%)$ & 0.99 & $0.65(95 \% \mathrm{Cl} 0.21$ to 1.0$)$ \\
\hline Cervical & $7 / 14(50 \%)$ & $8 / 14(57 \%)$ & 0.72 & $0.29(95 \% \mathrm{Cl}-0.21$ to 0.78$)$ \\
\hline Abdominal/pelvic & $1 / 14(7.1 \%)$ & $5 / 14(36 \%)$ & 0.077 & $0.32(95 \% \mathrm{Cl}-0.18$ to 0.81$)$ \\
\hline \multicolumn{5}{|l|}{ Heart } \\
\hline Left ventricle & $6 / 14(43 \%)$ & $7 / 14(50 \%)$ & 0.72 & $0.86(95 \%$ Cl 0.59 to 1.0$)$ \\
\hline Right ventricle & $2 / 14(14 \%)$ & $4 / 14(29 \%)$ & 0.38 & $0.59(95 \%$ Cl 0.11 to 1.0$)$ \\
\hline \multicolumn{5}{|c|}{ Central nervous system } \\
\hline Intracranial & $1 / 14(7.1 \%)$ & $0 / 14(7.1 \%)$ & 0.33 & $0.0(95 \%$ Cl 0.0 to 0.0$)$ \\
\hline Spinal/paraspinal & $1 / 14(7.1 \%)$ & $1 / 14(7.1 \%)$ & 0.99 & $1.0(95 \% \mathrm{Cl} 1.0$ to 1.0$)$ \\
\hline \multicolumn{5}{|l|}{ Lung parenchyma } \\
\hline- & $5 / 14(36 \%)$ & $5 / 14(36 \%)$ & 0.99 & 0.69 (95\% Cl 0.29 to 1.0$)$ \\
\hline \multicolumn{5}{|l|}{ Salivary glands } \\
\hline- & $0 / 14(0 \%)$ & $1 / 14(7.1 \%)$ & 0.33 & $-0.11(95 \% \mathrm{Cl}-0.26$ to 0.04$)$ \\
\hline \multicolumn{5}{|l|}{ Spleen } \\
\hline- & $0 / 14(0 \%)$ & $1 / 14(7.1 \%)$ & 0.33 & $0.0(-)$ \\
\hline \multicolumn{5}{|l|}{ Liver } \\
\hline- & $0 / 14(0 \%)$ & $0 / 14(0 \%)$ & - & - \\
\hline \multicolumn{5}{|l|}{ Bone } \\
\hline- & $0 / 14(0 \%)$ & $0 / 14(0 \%)$ & - & - \\
\hline \multicolumn{5}{|c|}{$\begin{array}{l}\text { Average number of sites/per subject with } \\
\text { active disease }\end{array}$} \\
\hline- & $1.6(1.0)$ & $1.8(1.3)$ & 0.75 & $0.78(95 \% 0.45$ to 0.93$)$ \\
\hline
\end{tabular}

FLT-PET = 3'-deoxy-3'-[ ${ }^{18}$ F]fluorothymidine positron emission tomography, FDG-PET = 2-deoxy-2-[18F]fluoro-D-glucose positron emission tomography

+ Agreement between categorical and continuous variables was assessed using Cohen's kappa and the intraclass correlation coefficient, respectively

between the various SUV measures on FLT and FDG was poor.

Of the 12 subjects with CS, the combination of perfusion/FLT-PET and perfusion/FDG-PET was positive in $11 / 12(92 \%)$ cases [ $\kappa=1.0(95 \%$ CI $1.0-1.0)]$. Interobserver agreement for active cardiac involvement was excellent for both FLT- and FDG-PET (FLT: $\kappa=0.85(95 \%$ CI $0.57-1.0$ ); FDG: $\kappa=0.72$ (95\% CI $0.38-1.0)$, Additional file 2: Table S2).

\section{Neurosarcoidosis findings}

A single case of NS was positive on both FLT and FDG (subject \#4); however, FLT-PET was able to identify two paraspinal (Fig. 7) and one intracalvarial lesions (Fig. 1), while FDG-PET only detected the paraspinal lesions. The agreement between FLT-PET and FDG-PET for the diagnosis of active NS was excellent $[\kappa=1.0(95 \%$ CI 1.0-1.0)] (Additional file 3: Table S3).

\section{Discussion}

In this prospective study, FLT-PET demonstrated excellent imaging and diagnostic properties for the evaluation of extra-cardiac sarcoidosis, as well as CS. FLT-PET was able to detect multiple NS lesions in a single subject with known neural involvement.

Limitations inherent in cardiac FDG-PET imaging are well-known and include extensive patient preparation, variable success in suppression of myocardial activity, and the existence of normal patterns of myocardial uptake complicating the interpretation of studies [10]. In particular, interpretation of the various cardiac uptake patterns is associated with only moderate inter-observer agreement [17]. Due to high, non-suppressible physiological uptake seen in the central nervous system (CNS), FDG-PET plays very little role in the evaluation of NS, with the extant literature limited to case reports. Because of these limitations, there is a need for a radiotracer which can accurately assess not only the lung parenchyma and lymph nodes, but also the myocardium and CNS, without the need for extensive patient preparation.

FLT offers multiple advantages, especially for the assessment of CS and NS. In particular, lack of physiological myocardial uptake obviates the need for extensive patient preparation resulting in increased patient convenience, and decreased risk of non-diagnostic studies due to patient non-compliance with 


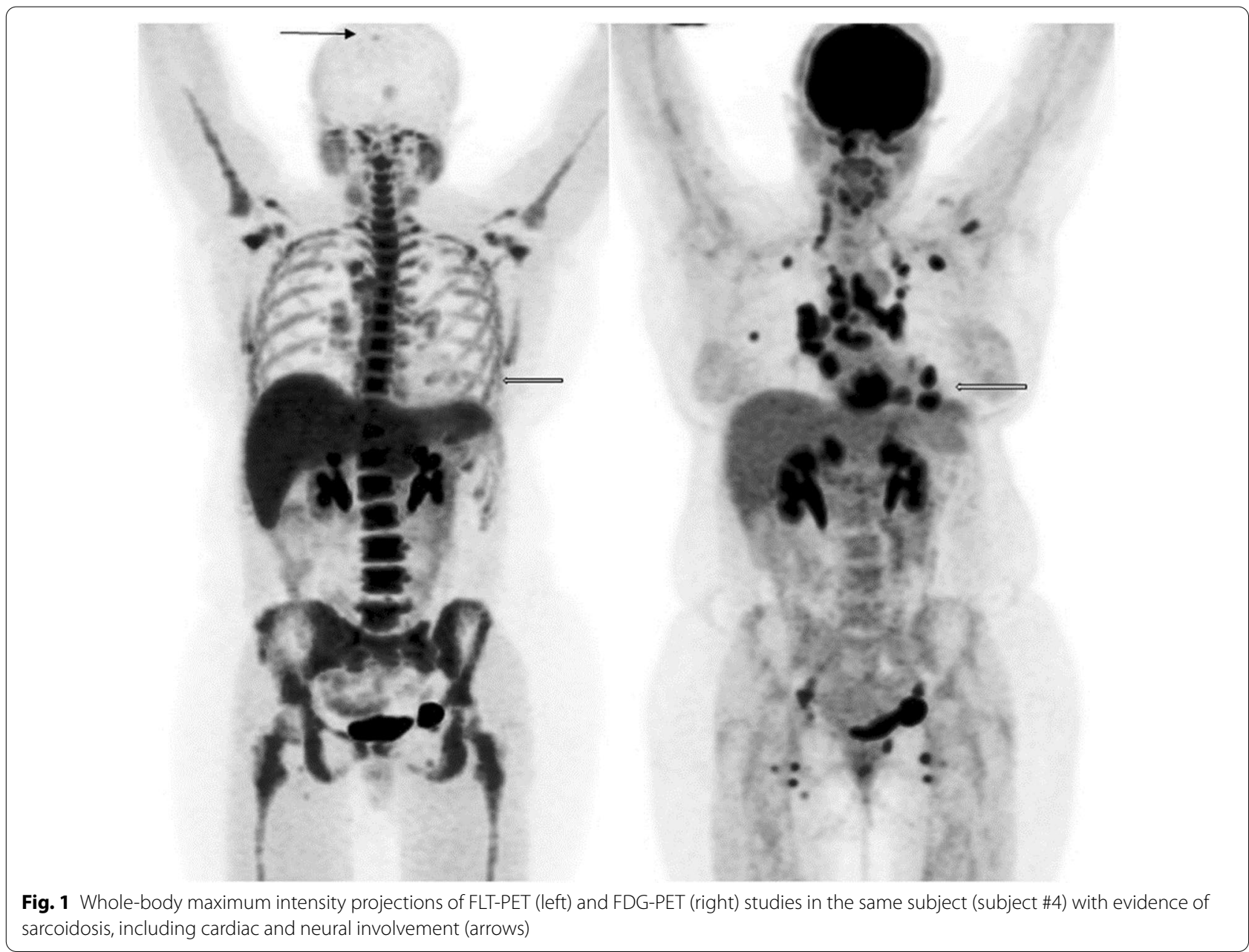

Table 3 Comparison of FLT- and FDG-PET for the diagnosis of active sarcoidosis $[K=0.76(95 \% \mathrm{Cl} 0.32-1.0)]$

\begin{tabular}{llll}
\hline & FLT-PET & Total \\
\cline { 2 - 3 } & $\begin{array}{l}\text { Positive scan consistent } \\
\text { with active sarcoidosis }\end{array}$ & $\begin{array}{l}\text { Negative } \\
\text { scan }\end{array}$ & \\
\hline FDG-PET & & 0 & 11 \\
$\begin{array}{l}\text { Positive scan consistent } \\
\text { with active sarcoidosis }\end{array}$ & 11 & 2 & 3 \\
Negative scan & 1 & 2 & 14 \\
Total & 12 & & \\
\hline
\end{tabular}

pre-imaging preparation instructions. Unlike FDG, there are no known normal variants demonstrating non-pathological myocardial FLT uptake, simplifying cardiac interpretation while lowering the risk of false-positive studies. In particular, our subject with a normal variant (i.e., lateral wall FDG uptake) had an unremarkable cardiac FLT study. In our subject with NS, both FLT and FDG were able to detect paraspinal

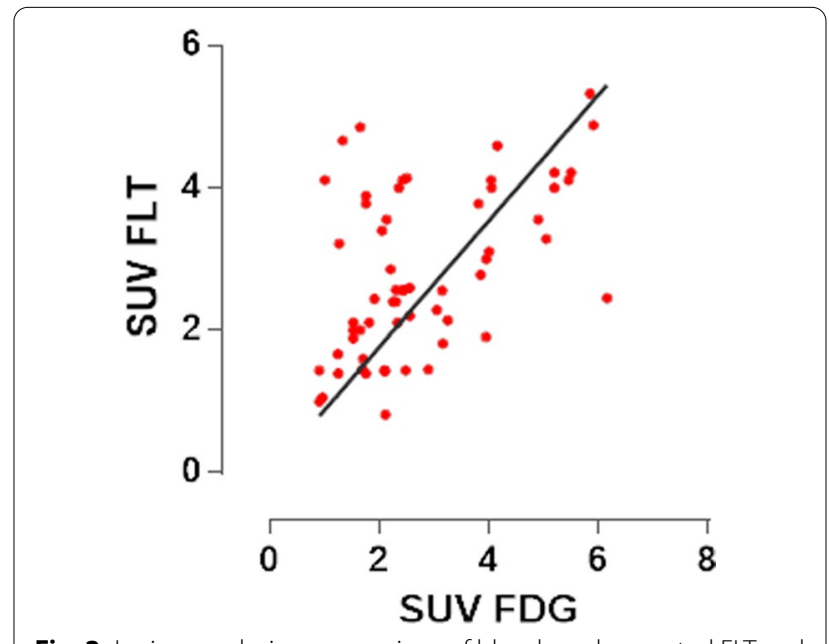

Fig. 2 Lesion-per-lesion comparison of blood-pool corrected FLT and FDG SUVs. The linear regression equation was $Y=0.89^{*} X$ 


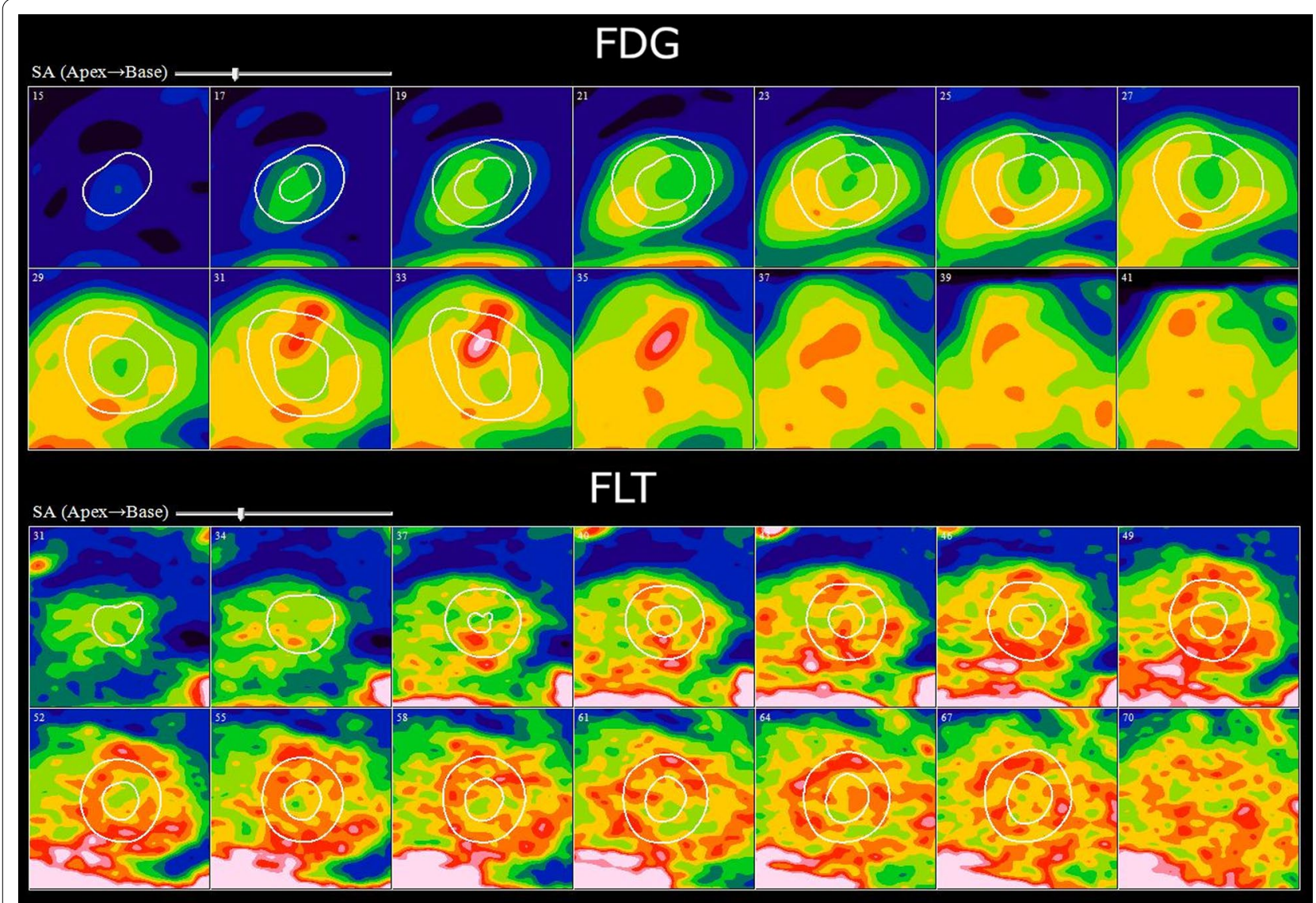

Fig. 3 Cardiac short axis views of subject \#10 demonstrating a focus of myocardial uptake in the basal anterior wall on FDG-PET (top) without corresponding uptake on the FLT-PET study (bottom). The diffuse heterogeneous uptake seen on the FLT study is in keeping with background blood pool uptake

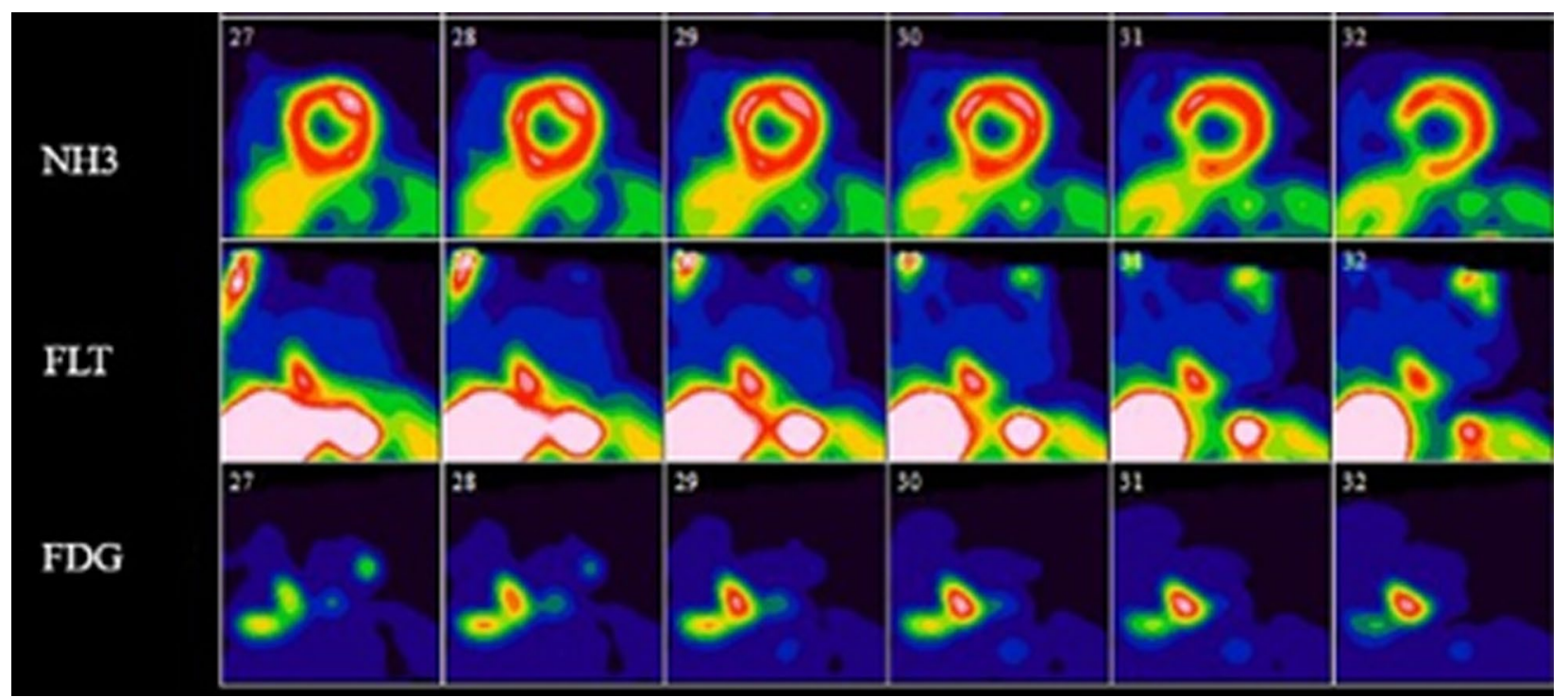

Fig. 4 Selected short axis images of cardiac perfusion PET (top), FLT (middle), and FDG (bottom) studies in short, vertical, and horizontal long axes in a single subject with CS. The findings were consistent with active CS on both the FLT and FDG studies. The images show the relationship between an area of scarring in the inferior aspect of the ventricular septum which overlaps an area of concordant uptake on both FDG and FLT 


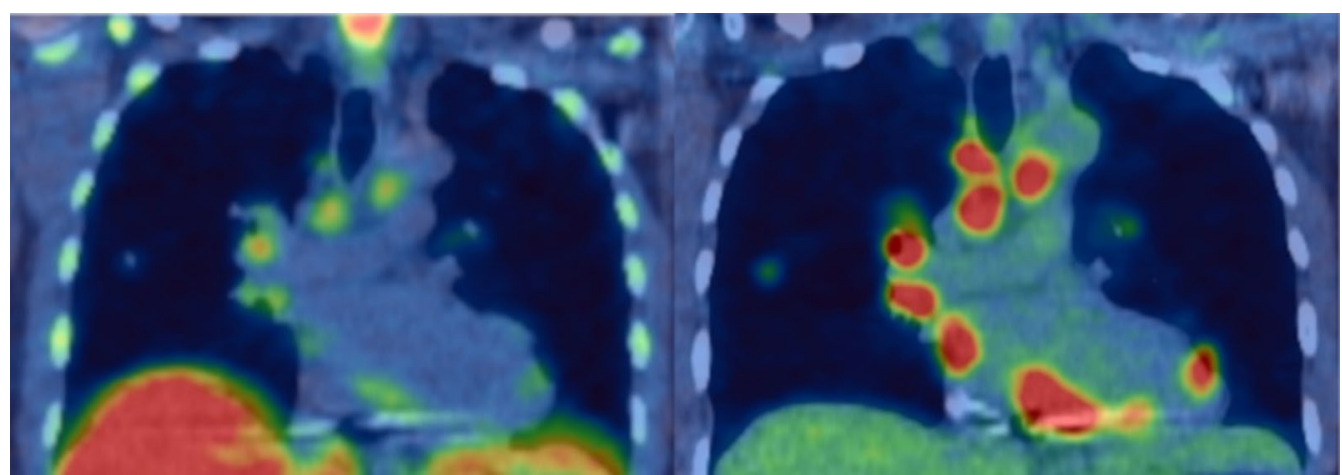

Fig. 5 Coronal section through the mediastinum demonstrating thoracic lymphadenopathy and cardiac involvement in a single subject on FLT-PET (left) and FDG-PET (right)

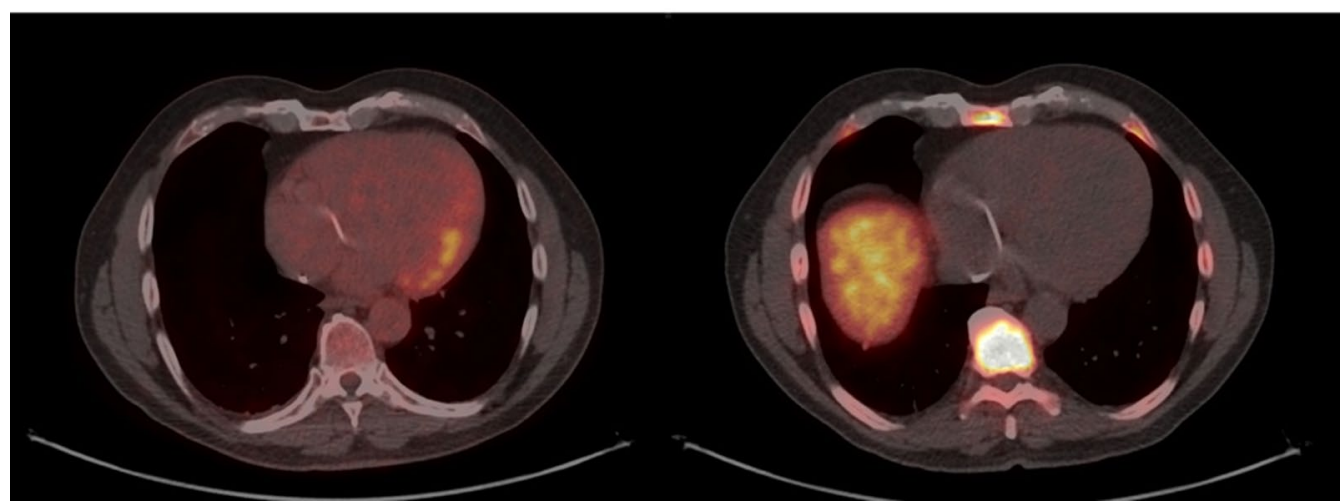

Fig. 6 Subject demonstrating isolated diffuse lateral ventricular wall FDG uptake (left), a finding usually interpreted as a normal variant. No corresponding uptake was present on the FLT study (right)

lesions; however, only FLT adequately demonstrated intracalvarial disease, due to a lack of background cerebral uptake. The high concordance in the distribution of lesions seen on FLT and FDG at key sites (i.e., heart, CNS, lung parenchyma), as well as for the overall number of organs involved, suggests a comparable performance to FDG disease staging. Furthermore, FLT effectively identified small pathological lymph nodes which could be used to identify active sites of disease for diagnostic biopsy. FLT showed a non-significant trend towards lower sensitivity for the detection of abdominal lymph nodes compared to FDG-this may have be related to the observation that, in many patients, involved lymph nodes are commonly seen around the porta hepatis and the high hepatic uptake seen on FLT may make identification of lymph 
Table 4 Summary of cardiac PET findings in subjects with CS $(N=12 *)$

\begin{tabular}{llll}
\hline & FLT-PET & FDG-PET & p value \\
\hline $\begin{array}{l}\text { \# of subjects with cardiac uptake } \\
\text { (\%) }\end{array}$ & $6(50 \%)$ & $7(58 \%)$ & 0.70 \\
\# of LV segments involved \pm SD & $6.2 \pm 1.5$ & $7.3 \pm 3.5$ & 0.50 \\
\# of segments in LAD ${ }^{\dagger}$ terri- & $2.5 \pm 1.0$ & $2.7 \pm 1.3$ & 0.75 \\
$\quad$ tory \pm SD & & & \\
\# of segments in LCX territory \pm SD & $1.3 \pm 1.0$ & $1.7 \pm 1.4$ & 0.59 \\
\# of segments in RCA territory \pm SD & $2.5 \pm 0.8$ & $2.9 \pm 1.5$ & 0.61 \\
\# of subjects with RV uptake (\%) & $2(17 \%)$ & $4(33 \%)$ & 0.17 \\
Maximum LV SUV \pm SD & $15.4 \pm 4.4$ & $13.7 \pm 6.0$ & 0.58 \\
LV SUV Total \pm SD & $27.7 \pm 8.4$ & $45.7 \pm 33.1$ & 0.22 \\
Maximum RV SUV $\pm S D$ a & $2.4 \pm 0.4$ & $4.6 \pm 5.0$ & 0.59 \\
& Perfusion PET & & \\
\# of subjects with perfusion & $10(83 \%)$ & & \\
$\quad$ defects (\%) & & & \\
SRS \pm SD & $6.2 \pm 4.6$ & & \\
SRS in LAD territory \pm SD & $3.2 \pm 2.9$ & & \\
SRS in LCX territory $\pm S D$ & $1.0 \pm 1.3$ & & \\
SRS in RCA territory \pm SD & $2.0 \pm 1.9$ & & \\
\hline
\end{tabular}

$L V$ left ventricle, $R V$ right ventricle, $L A D$ left anterior descending artery, $L C X$ left circumflex artery, $R C A$ right coronary artery, SUV standardized uptake value *Only subjects satisfying the HRS criteria $(N=12 / 14)$ were included in this analysis

† Vascular territories defined as per AHA 17 segment model [13]

a Includes only those subjects with evidence of RV uptake

Table 5 Concordance analysis of cardiac findings between FLT and FDG-PET in subjects satisfying the HRS criteria for CS $(N=12)$

\begin{tabular}{lc}
\hline & \multicolumn{1}{c}{ Agreement $^{\dagger}$} \\
\hline Subjects with LV uptake & $0.81(95 \% \mathrm{Cl} 0.47$ to 1.0$)$ \\
Subjects with RV uptake & $0.57(95 \% \mathrm{Cl} 0.08$ to 1.0$)$ \\
\# of LV segments involved & $0.81(95 \% \mathrm{Cl} 0.49$ to 0.94$)$ \\
\# of segments in LAD territory & $0.81(95 \% \mathrm{Cl} 0.51$ to 0.94$)$ \\
\# of segments in LCX territory & $0.54(95 \% \mathrm{Cl} 0.03$ to 0.83$)$ \\
\# of segments in RCA territory & $0.84(95 \% \mathrm{Cl} 0.56$ to 0.95$)$ \\
Maximum LV SUV & $0.22(95 \% \mathrm{Cl}-0.38$ to 0.69$)$ \\
Maximum RV SUV & $0.36(95 \% \mathrm{Cl}-0.24$ to 0.76$)$ \\
Mean segmental SUV & $-0.002(95 \% \mathrm{Cl}-0.58$ to 0.57$)$ \\
LV SUV $_{\text {Total }}$ & $0.20(95 \% \mathrm{Cl}-0.42$ to 0.70$)$
\end{tabular}

$\dagger$ Agreement between categorical and continuous variables was assessed using Cohen's kappa and the intraclass correlation coefficient, respectively

nodes at the porta challenging; however, further studies will needed to confirm this. Although not shown in our study, FLT-PET could, in theory, be less sensitive than FDG-PET for the detection of sarcoidosis lesions within the liver and bone marrow due to the significant uptake normally seen in these organs; however, this

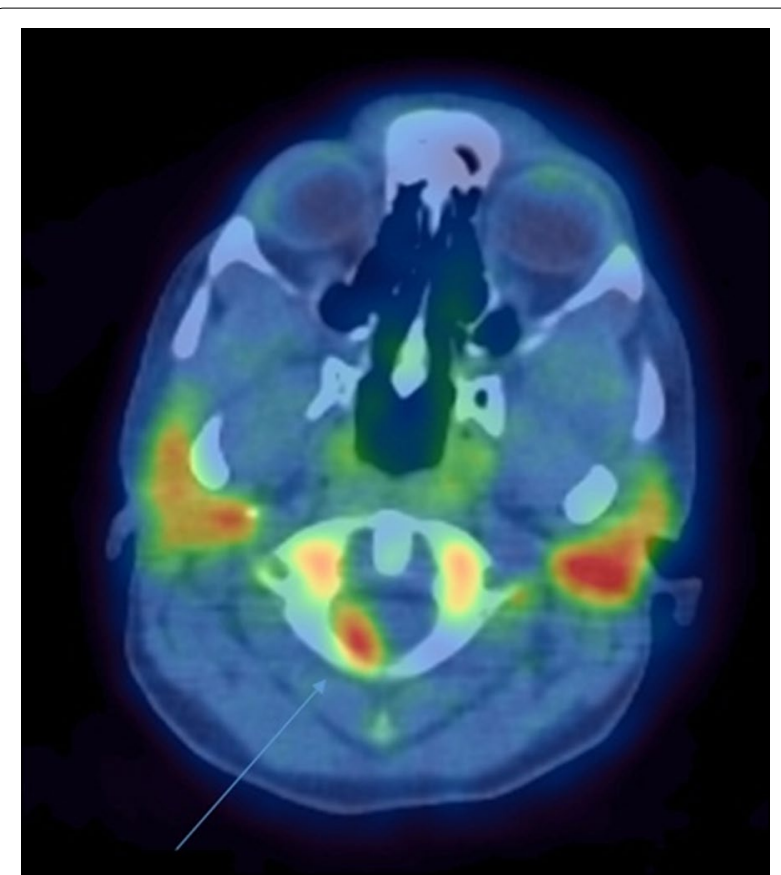

Fig. 7 FLT-PET study in a subject with neurosarcoidosis (arrow) (subject \#4)

may deemed acceptable in light of the improved assessment of the heart and CNS and the relatively limited clinical impact of hepatic and marrow involvement.

Our study was not designed to determine the utility of FLT lung uptake as a therapeutic target; however, one would expect that, in order to duplicate the success of FDG in this respect, there should at least be good concordance between FLT and FDG for the assessment of parenchyma involvement, which was present in our results. Additional studies will be needed to assess the effectiveness of FLT-PET in the assessment of therapeutic response.

Although FLT demonstrates a lower degree of uptake (SUV) in sarcoidosis lesions when compared to FDG, the difference was not clinically significant with lesions demonstrating similar signal to background with both tracers. After correction for background activity (i.e., blood pool) the difference in SUVs was not statistically significant. Linear regression analysis revealed a relatively low degree of correlation between FDG and FLT uptake compatible with our previously reported results [12]. This can be attributed to the fact that the degree of FDG uptake is non-specific and reflects all underlying metabolic processes while FLT uptake is specific to proliferative activity.

FLT activity is expected to represent granuloma burden while FDG reflects general inflammatory activity. Differences in uptake mechanisms between FLT and 
FDG could be useful for disease monitoring and therapy assessment. In particular, we speculate that the specificity of the uptake mechanism of FLT may be particularly well-suited for monitoring response to therapy; however, additional studies are needed.

It is unclear why a single subject (\#10) showed discordant myocardial findings (positive on FDG, negative on FLT) while another subject (\#8) showed discordant whole-body FLT and FDG findings (positive on FLT, negative on FDG). In both cases, these subjects were undergoing treatment at the time of imaging. It may be that the discordance was related to treatment effects. Also, it should be noted that the imaging findings in both cases were subtle and the discordances might be attributable to limited sensitivity (i.e., false-negative) and/or specificity (i.e., false-positive) for either FLT or FDG.

Our results can be compared to those of Norikane et al. [11] who conducted a retrospective study involving 20 subjects with newly diagnosed cardiac sarcoidosis and compared FLT and FDG-PET findings. They found that both FLT and FDG had high accuracy for cardiac and extra-cardiac thoracic sarcoidosis. There are, however, several important differences between our studies. Our study was prospective and used the HRS criteria as gold standard, compared to the JMHW criteria used by Norikane et al. Furthermore, subjects undergoing cardiac evaluation in the Norikane study had a high rate of inconclusive FDG studies (4/20) while all of our subjects' studies were diagnostic. Also, Norikane limited their imaging to the thorax while we acquired near whole-body images, which enabled us to compare findings in extra-thoracic sarcoidosis (including intracalvarial NS). Importantly, the work by Norikane et al. did not incorporate cardiac perfusion imaging in their analysis - we have previously shown significant associations between the findings on FLT-PET and perfusion imaging [12]. Finally, none of their subjects had evidence of NS.

Some limitations of this study should be acknowledged. Our sample size was small, and lack of negative controls prevented us from calculating the accuracy for wholebody sarcoidosis. In order to remedy this, a larger prospective study is underway. In addition, all subjects were referred for suspicion of CS. This subgroup of subjects may not be representative of typical subjects with sarcoidosis and may have influenced the distribution of sarcoidosis lesions we observed in our study. Finally, there was only a single subject with a clinical diagnosis of NS, limiting our assessment of the utility of FLT-PET for neural involvement.

In summary, FLT-PET has the ability to accurately detect sarcoidosis lesions, including cardiac and CNS involvement, without the need for extensive patient preparation. Follow-up studies are ongoing in order to confirm these findings.

\section{Supplementary information}

Supplementary information accompanies this paper at https://doi. org/10.1186/s13550-020-00742-x.

Additional file 1. Table S1: Comparison of both readers' overall interpretation of the FLT- and FDG-PETs for active sarcoidosis [FLT $K=0.81(95 \% \mathrm{Cl}$ $0.46-1.0)$, FDG $K=1.0(95 \%$ Cl 1.0-1.0)].

Additional file 2. Table S2: Comparison of both readers' overall interpretation of the FLT- and FDG-PETs for cardiac involvement [FLT $K=0.85$ (95\% Cl 0.57-1.0), FDG $K=0.72$ (95\% Cl 0.38-1.0)].

Additional file 3. Table S3: Comparison of both readers' overall interpretation of the FLT- and FDG-PETs for neural involvement [FLT $K=1.0(95 \% \mathrm{Cl}$ 1.0-1.0), FDG $K=1.0(95 \% \mathrm{Cl} 1.0-1.0)$ ].

\section{Abbreviations}

CNS: Central nervous system; CS: Cardiac sarcoidosis; CT: Computed tomography; ECG: Electrocardiogram; FDG: 2-Deoxy-2-[18 F]fluoro-D-glucose; FLT: $3^{\prime}$-Deoxy-3'-[18 F]fluorothymidine; HRS: Heart Rhythm Society; ICC: Intraclass correlation coefficient; JMHW: Japanese Ministry of Health and Welfare; Kg: Kilogram; LV: Left ventricular; MBq: Megabecquerel; $\left[{ }^{13} \mathrm{~N}_{\mathrm{NH}}:{ }^{13} \mathrm{~N}\right.$-ammonia; NS: Neurosarcoidosis; PET: Positron emission tomography; ${ }^{82} \mathrm{Rb}$ : Rubidium-82; SUV: Standardized uptake value.

\section{Acknowledgements}

Not applicable.

\section{Authors' contributions}

PM, MPG, DJ, EL, PBN, RdK, RSB, and DB drafted the manuscript. PM, DJ, PBN and $D B$ were involved in data acquisition. PM, MPG, RdK, DB were involved in analysis. PM, RSB, DB were involved in study design. RSB analyzed the data. All authors read and approved the final manuscript.

\section{Funding}

The work has been supported in part by a research trial grant from the Ministry of Health and Long Term Care Research (Grant \# 06374) for Ontario PET Cardiac Sarcoidosis Trial, and by a UOHIAMO Innovation grant from the University of Ottawa Heart Institute. The project is in collaboration with the Ontario Cardiac PET Special Access Program and the PET Steering Committee of Ontario overseen by Cancer Care Ontario. This work has also been partially supported by the Canadian Institute of Health Research (D. Birnie, Pl; R. Beanlands COPI) for The Cardiac Sarcoidosis Cohort Study (CHASM-CS). (Grant Nos. 342139, NCT01477359). No potential conflicts of interest relevant to this article exist.

\section{Availability of data and materials}

The datasets used and/or analyzed during the current study are available from the corresponding author on reasonable request.

\section{Ethics approval and consent to participate}

Ethics approval for this study was granted by The University of Ottawa Heart Institute ethics board. All subjects provided informed consent prior to participating.

\section{Consent for publication}

Consent to publish was obtained.

\section{Competing interests}

None.

\section{Author details}

${ }^{1}$ Functional Imaging Department, BC Cancer Agency, 600 W 10th Ave, Vancouver, BC V5Z 4E6, Canada. ${ }^{2}$ Gordon Center for Medical Imaging, Massachusetts General Hospital, Harvard Medical School, Boston, MA, USA. ${ }^{3}$ Department of Medical Imaging, Montreal Heart Institute, Université de Montréal, 
Montreal, QC, Canada. ${ }^{4}$ Centre Hospitalier de L'Université de Montréal (CHUM), Université de Montréal, Montreal, QC, Canada. ${ }^{5}$ Molecular Function and Imaging Program, The National Cardiac PET Centre and the Arrhythmia Service, Division of Cardiology, Department of Medicine, University of Ottawa Heart Institute, Ottawa, Canada. ${ }^{6}$ Division of Nuclear Medicine, Department of Medicine, The Ottawa Hospital, Ottawa, ON, Canada. ${ }^{7}$ Arrhythmia Service, Division of Cardiology, Department of Medicine, University of Ottawa Heart Institute, 40 Ruskin Street - Room H 1285, Ottawa, ON K1Y 4W7, Canada.

Received: 9 August 2020 Accepted: 25 November 2020

Published online: 10 December 2020

\section{References}

1. Iwai K, Sekiguti M, Hosoda Y, DeRemee R, Tazelaar H, Sharma O, et al. Racial difference in cardiac sarcoidosis incidence observed at autopsy. Sarcoidosis. 1994;11:26-31.

2. James DG. Life-threatening situations in sarcoidosis. Sarcoidosis Vasc Diffuse Lung Dis Off J WASOG. 1998;15:134-9.

3. Hoitsma E, Faber CG, Drent M, Sharma OP. Neurosarcoidosis: a clinical dilemma. Lancet Neurol. 2004;3:397-407.

4. Iwai K, Tachibana T, Takemura T, Matsui Y, Kitaichi M, Kawabata Y. Pathological studies on sarcoidosis autopsy. I. Epidemiological features of 320 cases in Japan. Acta Pathol Jpn. 1993:43:372-6.

5. Teirstein AS, Machac J, Almeida O, Lu P, Padilla ML, lannuzzi MC. Results of 188 whole-body fluorodeoxyglucose positron emission tomography scans in 137 patients with sarcoidosis. Chest. 2007;132:1949-53.

6. Braun JJ, Kessler R, Constantinesco A, Imperiale A. 18F-FDG PET/CT in sarcoidosis management: review and report of 20 cases. Eur J Nucl Med Mol Imaging. 2008;35:1537.

7. Kaira K, Ishizuka T, Yanagitani N, Sunaga N, Hisada T, Mori M. Value of FDG positron emission tomography in monitoring the effects of therapy in progressive pulmonary sarcoidosis. Clin Nucl Med. 2007;32:114-6.

8. Nishiyama Y, Yamamoto Y, Fukunaga K, Takinami H, Iwado Y, Satoh K, et al. Comparative evaluation of 18F-FDG PET and 67Ga scintigraphy in patients with sarcoidosis. J Nucl Med. 2006:47:1571-6.

9. Youssef G, Leung E, Mylonas I, Nery P, Williams K, Wisenberg G, et al. The use of 18F-FDG PET in the diagnosis of cardiac sarcoidosis: a systematic review and metaanalysis including the Ontario experience. J Nucl Med. 2012;53:241-8.

10. Chareonthaitawee P, Beanlands RS, Chen W, Dorbala S, Miller EJ, Murthy $\mathrm{VL}$, et al. Joint SNMMI-ASNC expert consensus document on the role of 18F-FDG PET/CT in cardiac sarcoid detection and therapy monitoring. J Nucl Med. 2017;58:1341-53.

11. Norikane T, Yamamoto Y, Maeda Y, Noma T, Dobashi H, Nishiyama Y. Comparative evaluation of 18F-FLT and 18F-FDG for detecting cardiac and extra-cardiac thoracic involvement in patients with newly diagnosed sarcoidosis. EJNMMI Res. 2017;7:69.

12. Martineau P, Pelletier-Galarneau M, Juneau D, Leung E, Nery P, de Kemp $R$, et al. Diagnosing cardiac sarcoidosis with FLT PET: a prospective controlled pilot study. JACC: Cardiovasc Imaging. 2019;12(11):2280-1.

13. Cerqueira MD, Weissman NJ, Dilsizian V, Jacobs AK, Kaul S, Laskey WK, et al. Standardized myocardial segmentation and nomenclature for tomographic imaging of the heart: a statement for healthcare professionals from the cardiac imaging committee of the council on clinical cardiology of the American Heart Association. Circulation. 2002;105:539-42.

14. Birnie DH, Sauer WH, Bogun F, Cooper JM, Culver DA, Duvernoy CS, et al. HRS expert consensus statement on the diagnosis and management of arrhythmias associated with cardiac sarcoidosis. Heart Rhythm. 2014;11:1304-23.

15. McGraw KO, Wong SP. Forming inferences about some intraclass correlation coefficients. Psychol Methods. 1996;1:30.

16. Cicchetti DV. Guidelines, criteria, and rules of thumb for evaluating normed and standardized assessment instruments in psychology. Psychol Assess. 1994;6:284-90.

17. Ohira H, Ardle BM, deKemp RA, Nery PB, Juneau D, Renaud JM, et al. Inter- and intra- observer agreement of FDG-PET/CT image interpretation in patients referred for assessment of cardiac sarcoidosis. J Nucl Med. 2017;58:1324-9.

\section{Publisher's Note}

Springer Nature remains neutral with regard to jurisdictional claims in published maps and institutional affiliations.

\section{Submit your manuscript to a SpringerOpen ${ }^{\circ}$ journal and benefit from:}

- Convenient online submission

- Rigorous peer review

- Open access: articles freely available online

- High visibility within the field

- Retaining the copyright to your article

Submit your next manuscript at springeropen.com 